\title{
A Wave Emulator for Ocean Wave Energy, A Froude-scaled Dry Power Take-Off Test Setup
}

\author{
Kristof L. De Koker ${ }^{\mathrm{a}, *}$, Guillaume Crevecoeur ${ }^{\mathrm{a}}$, Bart Meersman ${ }^{\mathrm{a}}$, Marc \\ Vantorre $^{\mathrm{b}}$, Lieven Vandevelde ${ }^{\mathrm{a}}$ \\ ${ }^{a}$ Department of Electrical Energy, Systems and Automation, Ghent University, \\ Technologiepark-Zwijnaarde 913, 9052 Ghent, Belgium \\ ${ }^{b}$ Maritime Technology Division, Ghent University, Technologiepark-Zwijnaarde 904, 9052 \\ Ghent, Belgium
}

\begin{abstract}
A dry laboratory environment has been developed to test Power Take-Off (PTO) systems for Wave Energy Converters. The costs accompanied by testing a wave energy converter and its PTO at sea are high due to the difficult accessibility of (remote) test locations. Next to easy accessibility, the lab setup provides controllable waves at a relatively lower cost. The setup enables extensive analysis of the dynamics of a PTO during its mechanical towards electrical energy conversion. The scaled setup is designed such that it resembles as close as possible the real system. Froudes similarity law provides easy transformation. The floater and waves are represented by a Wave Emulator, the motion of which is determined by a time series of the wave exciting forces supplemented with the actual hydrodynamic reaction forces due to the motions of the floater. A real-time calculation method is introduced, accounting for the actual PTO actions. Furthermore, the inertia of the floater is represented in the emulators rotary inertia, and a compensation method is proposed enabling an identical normalized PTO load curve as at full scale. Comparison between experimental and simulation results have been performed and good correlation between the movement of setup and simulations has been found.
\end{abstract}

Keywords: Ocean wave energy, Power take-off (PTO), renewable energy,

\footnotetext{
${ }^{*}$ Corresponding author

Email address: Kristof.DeKoker@UGent.be (Kristof L. De Koker)
} 
Froude scaling, wave emulator, PTO dry-testing

\section{1. Introduction}

The Wave Pioneer is a test Wave Energy Converter (WEC), designed and built during the FlanSea project [1, for wave energy conversion in a moderate wave climate. The Wave Pioneer is a point-absorber type [2 consisting of a buoy connected with the seabed by a cable. In the buoy, the cable is wound onto a drum and connected with two electrical machines through a gearbox as depicted in Fig. 1. The gearbox increases the working torque towards the drum, while decreasing the speed. The machines are connected to two variable frequency drives. This assembly of drum, gearbox, electrical machines and drives is referred to as the Power Take-Off (PTO) system, and converts the power absorbed from the waves to electrical energy. When the buoy is pushed upwards by the wave motion, the cable is wound off and electricity is generated by applying a braking torque by the electrical machines. During the downward movement, the electrical machines act as motor to wind up the cable and keep it under tension, and apply reactive control [3] to optimise the energy yield. This paper describes a lab setup that was conceived to assess the dynamics of the chosen PTO machines and to test the control strategy for the uptake of power.

Before testing a selected PTO technology in a wave energy converter at sea, a setup in a protected and controllable environment is advisable, as interventions at a remote location can be very expensive and impractical due to weather conditions. An intermediate step may consist of tests in a wave flume or tank. However, the (large) scale factor to be selected for such tests in general does not allow to investigate the behaviour of the electrical machines of which the PTO is composed. For example, in [4, 5, 6] wave basin experiments have been performed, for which the smallest length scale factor used was approximately 24 [7. Using Froude similarity this results in a scale factor of approximately 70000 for the power. Therefore, one might consider to reserve these tests for the study of the hydrodynamic behaviour of the floater and opt for a dry test bench for 


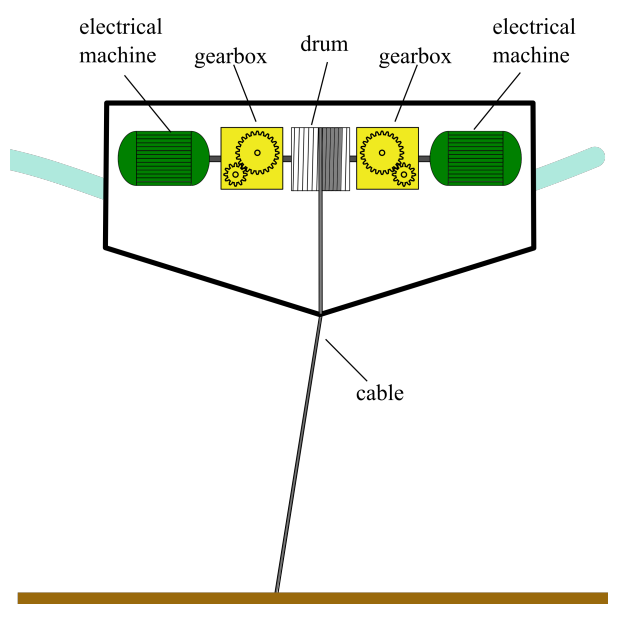

Figure 1: Schematic overview of the Wave Pioneer and its PTO system: two electrical machines are connected to a drum by means of a gearbox.

29 the PTO. A protected laboratory environment facilitates the feasibility study of a chosen PTO topology to meet the requirements of the highly dynamic movements.

The setup's design is primordial to obtain a realistic emulation and accurate testing and assessment of the energy conversion by acquiring the PTO's dynamic response to varying incoming waves. Moreover, control algorithms and the power take-off technology have a drastic influence on the energy conversion of the WEC [8]. For both hardware testing and control algorithm examination purposes, the design described in this paper aims at (a) maximum resemblance with a full scale wave energy converter, (b) performing tests in a dry setup and (c) testing at equal normalized load conditions as the full scale PTO in the buoy. The resemblance should encompass the main factors of the floater's movement: it moves due to the resulting force of hydrodynamic forces and the PTO force acting on the mass of the buoy. As a result of the motion, the buoy experiences hydrodynamic reaction forces. Hence, emulating the floater motion needs to consist of reproducing the relevant forces and masses. As described in [9], the reproduction of the WEC's inertia and hydrostatic restoring force are two key elements for a realistic lab setup. Herein, the focus lies on linear actuators and 
the inertia and restoring force are reproduced by means of the body of water in a U-tube. Other linear test rigs were described in [10, 11]. For the Pelamis, a scaled hydraulic PTO was introduced in [12. A rotary shaft-to-shaft connected setup was presented in [13]. In [14] tests were performed on a rotary setup for an oscillating-water-column wave energy converter. This setup was reused to test latching control on an oscillating-water-column device in [15], and for testing speed control strategies for an oil-hydraulic PTO in [16].

In contrast to 9 , the present article describes a dry setup where the physical behaviour of floater body and waves is represented by rotary inertias and an electromechanical torque. Compared to the linear PTO in [10, 11] and the hydraulic one in [12, a rotary electrical PTO is implemented to match with the chosen PTO topology of the Wave Pioneer. In [13], time series of torques are used, in contrast, in this work the hydrodynamic forces are calculated interactively in the emulation. Thus the applied torque of the emulator corresponds to the actual force acting on the body in irregular waves based on the actual floater motion also accounting for changing PTO actions. This is accomplished by real-time calculation of the restoring force and the non-linear term of the radiation force and has the advantage that the PTO action can be varied during the tests.

For the setup presented in this article, a shaft-to-shaft assembly of two electrical machines is proposed, they can act both as generator and as motor and are therefore further referred to as (electrical) machine. One machine acts as a Wave Emulator that applies a hydrodynamic force equivalent torque. Subsequently the scaled PTO machine, the shaft of which is connected directly to the emulator's shaft, can be controlled as if it were installed in a buoy at sea. To reduce the power of the setup, a scaling is implemented. Froude scaling [17, 18] is proposed as it enables transformation of the entire wave energy converter and accompanying hydrodynamic environment towards a scale model. 


\section{Mathematical models}

When hydrodynamic and PTO forces interact with a floating buoy, its movement depends on the magnitude of these forces and the mass of the buoy and rotary inertia of the PTO. To enhance the resemblance of the setup with reality, not only the forces but also the mass and rotary inertia should be reproduced. The rotary inertia of the PTO influences the motion of the floater as it contributes to the PTO force as an inertial force due to its acceleration. Especially when the PTO drive train contains a gearbox, because the equivalent inertia at the slow side of the gearbox is proportional to the square of the gear ratio.

To represent the floater and the PTO in the lab setup, the interaction between both needs to be examined, as well as the hydrodynamic forces. The following sections describe the equation of motion of the floater, the PTO model, and the hydrodynamic forces on the floater.

The top level of the model describing the motion of the wave energy converter is the equation of movement. Principally the floater is a mass $m$ with two forces working on it, $F_{\text {hyd }}$ and $F_{\mathrm{PTO}}$, as illustrated in Fig. 2 . The equation of motion is thus:

$$
m \frac{d^{2} z}{d t^{2}}=F_{\mathrm{hyd}}+F_{\mathrm{PTO}}
$$

\subsection{PTO model}

In Fig. 2, a schematic overview of the PTO in the floater is given. In the Wave Pioneer two machines were installed, though this was mainly for practical reasons, therefore all further explanation is done in the assumption of one machine. The electrical machine is connected through a gearbox to a drum. On the drum a cable is wound which is connected to the sea bottom. The torque on the shaft of the electrical machine is increased by the gearbox and subsequently translated to a force on the cable by the drum. The force on the cable is defined as the PTO force $F_{\text {PTO }}$ and can be expressed as

$$
F_{\mathrm{PTO}}=\frac{R_{\mathrm{g}}}{r} T_{\text {shaft }}
$$




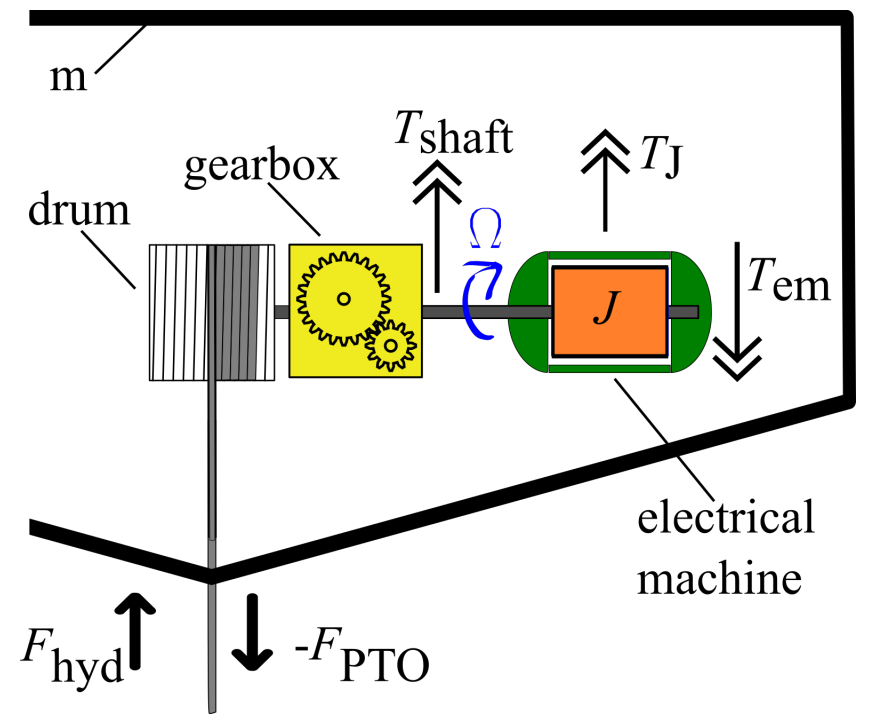

Figure 2: Schematic overview of the forces acting on the floater and the PTO in the floater

with $R_{\mathrm{g}}$ the gearbox ratio, $r$ the radius of the drum and $T_{\text {shaft }}$ the torque on the machine shaft. Taking the rotor inertia of the machine into account, the force can be expressed in relation to the electromagnetic torque $T_{\mathrm{em}}$ as

$$
F_{\text {PTO }}=\frac{R_{\mathrm{g}}}{r}\left(T_{\mathrm{em}}-T_{\mathrm{J}}\right)
$$

with $T_{\mathrm{J}}=-J d \Omega / d t$ the inertial torque of the rotor inertia and $\Omega$ the rotational speed of the shaft in radians per second. Internal friction in the machine, such as from the bearings, is neglected.

\subsection{Hydrodynamic model}

The resulting force of the water acting on the WEC is defined as the hydrodynamic force $F_{\text {hyd }}$. It can be described as the sum of the exciting wave force $F_{\text {ex }}$, the restoring force $F_{\text {res }}$, and the radiation force $F_{\text {rad }}$ :

$$
F_{\text {hyd }}=F_{\text {ex }}+F_{\text {res }}+F_{\text {rad }}
$$

The model assumes a buoy in pure heave motion, in a single degree of freedom. The following subsections describe the different terms of Equation (4). 


\subsubsection{Exciting wave force}

The exciting wave force is based on calculations with WAMIT and is the force that a body would experience if kept still in a passing wave, with the still water line at the still water level. This term of the hydrodynamic force is movement independent and is thus read from a time series of processed WAMIT ${ }^{\circledR}$ calculations per sea state (SS) for the given WEC using a JONSWAP spectrum. Six sea states were generated at scale for significant wave heights from $0.25 \mathrm{~m}$ to $2.75 \mathrm{~m}$ and mean periods of $3.6 \mathrm{~s}$ to $6.2 \mathrm{~s}$ at full scale.

\subsubsection{Hydrostatic restoring force}

The hydrostatic restoring force includes the Archimedes force $F_{\text {arch }}$ and the gravity force $F_{\mathrm{g}}$. It can also be expressed as the spring force in the mass-springdamper analogy for a WEC that is cylindrical around the waterline:

$$
F_{\text {res }}=F_{\text {arch }}-F_{\mathrm{g}}=\rho g\left[V(t)-V_{0}\right]=-k z
$$

The spring constant $k$ is therefore called the hydrostatic restoring coefficient and is expressed as $k=\rho g A_{\mathrm{w}}$, where $A_{\mathrm{w}}$ is the waterline area, $g$ the gravity constant, $\rho$ the density of the water, $z$ is the vertical position of the buoy relative to the still water line, $V(t)$ the instantaneous, submerged buoy volume and $V_{0}$ being the displacement volume at rest.

\subsubsection{Radiation force}

The radiation force $F_{\text {rad }}$ is defined as the hydrodynamic reaction force due to the motions of the floating body in still water. The radiation force can be formulated as

$$
F_{\mathrm{rad}}=-m_{a}(\omega) \frac{d^{2} z}{d t^{2}}-b_{h y d}(\omega) \frac{d z}{d t}
$$

in the frequency domain with $\omega$ the angular frequency of the wave and $b_{h y d}$ the hydrodynamic damping coefficient. However, a time domain approach is required due to the necessity of considering irregular seas and due to the nonlinearities induced by the cable and the control strategy. In the time domain, 
the radiation force $F_{\text {rad }}$ can be decomposed in a linear added mass term and a convolution product [19, 20]:

$$
\begin{aligned}
& F_{\mathrm{rad}}=F_{\mathrm{rad}, 1}+F_{\mathrm{rad}, 2} \\
& F_{\mathrm{rad}, 1}=-m_{a, \infty} \frac{d^{2} z}{d t^{2}}
\end{aligned}
$$

$$
F_{\mathrm{rad}, 2}=-\int_{0}^{t} K_{r}(t-\tau) \frac{d z(\tau)}{d t} d \tau
$$

where $m_{a, \infty}$ is the infinite frequency limit of the added mass, it is defined as the factor by which the buoy's vertical acceleration has to be multiplied to obtain the acceleration dependent component of the hydrodynamic reaction force, and can therefore be interpreted as the mass of the water surrounding the buoy and moving along with it. $K_{r}$ is the radiation impulse response function obtained by Fourier transformation of the frequency domain hydrodynamic parameters of added mass and damping, which can be computed with frequency domain Boundary Element Method codes like WAMIT. $K_{r}$ is obtained from [20]:

$$
K_{r}(t)=\frac{2}{\pi} \int_{0}^{\infty} b_{h y d}(\omega) \cos (\omega) d \omega
$$

The frequency to time domain utility provided by WAMIT has been used to determine $K_{r}$. Duclos and Clément et al. 21] developed a method to result in a system of ordinary differential equations which are implemented in the calculation of this component of the hydrodynamic force.

\subsubsection{Importance of real-time emulation of the hydrodynamic force}

The movement of a floating body is initially induced by the exciting wave force, but its inertia and the PTO force acting on the body influence the magnitude of the hydrodynamic force significantly, mainly due to the increasing restoring force as a result of the movement. To illustrate the influence of the movement on the total hydrodynamic force, the different calculated hydrodynamic force components have been extracted from simulations and are plotted in Fig. 3. From this graph it can be noticed that the total hydrodynamic force 


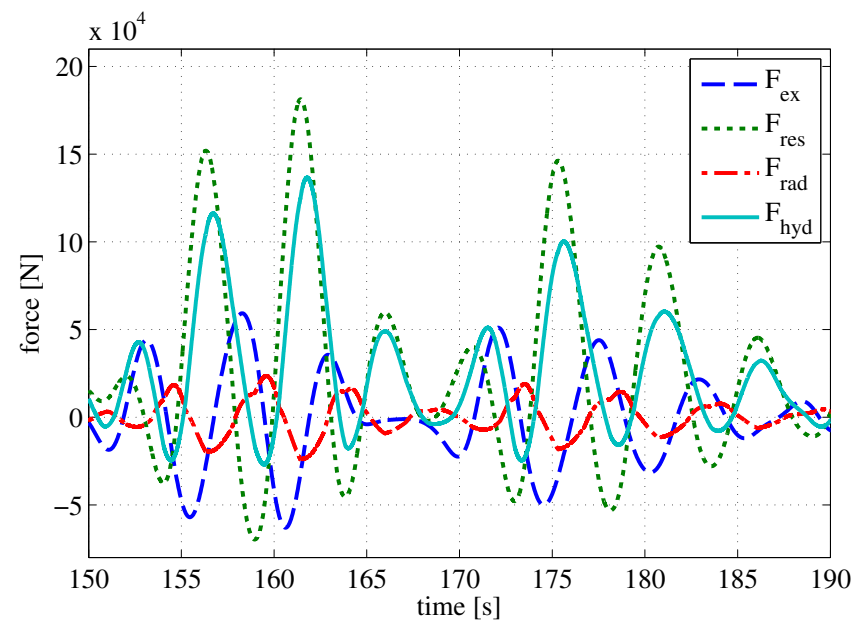

Figure 3: Plot of the total hydrodynamic force and its components for a control consisting of phase control

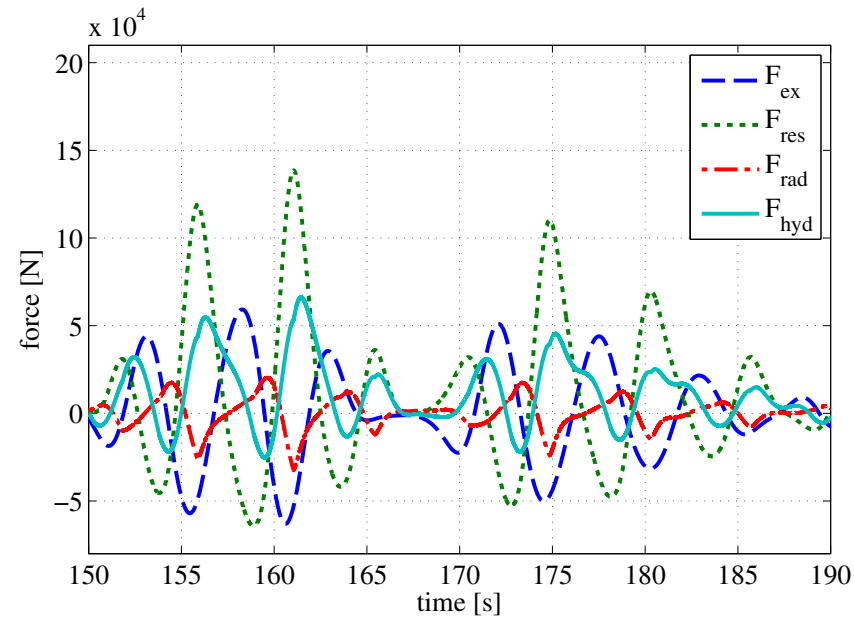

Figure 4: Plot of the total hydrodynamic force and its components for a control without phase control 
peaks at three times the peak value of the exciting force. It emphasizes the importance of including the different force components of Equation (4) in the control of the Wave Emulator, and not only the exciting wave force. The resulting movement of a body exposed to only the exciting wave force would differ greatly from the movement of a body subjected to all resulting hydrodynamic forces.

Additionally, the magnitude of the PTO action has a significant influence on the resulting hydrodynamic force. As an example, a second simulation has been done with different control parameters than the first simulation for Fig. (3). Following a control algorithm described in [3], the first simulation uses phase control, the second simulation is done without phase control. The results of the latter are plotted in Fig. 4. The effect of the changing PTO action is illustrated by the difference between the total hydrodynamic force $F_{\text {hyd }}$ of Fig. 3 and Fig. 4. While both simulations used the same exciting wave force $F_{\mathrm{ex}}$ (dashed blue line), it is clearly visible that the total hydrodynamic force $F_{\text {hyd }}$ (solid cyan line) is much lower for the second case.

It is concluded that, to have a setup that represents reality and that can react on changing PTO actions, a real-time calculated hydrodynamic force is crucial to control the wave emulator.

\section{Lab setup}

\subsection{Representation of the floater and sea in the setup}

As the intended layout of the setup is a shaft-to-shaft connection of two electrical machines, the wave emulator machine needs to provide the identical torque to the shaft of the PTO machine as in a real wave energy converter. Thus the floater and the sea need to have an equivalent representation at the PTO machine shaft.

The floater in the water can be seen as an inertial mass with two forces acting on it, the hydrodynamic force and the PTO force. The wave emulator machine has a rotary inertia where two torques act upon, the electromagnetic torque 
and the torque from the shaft. Therefore it is proposed that the hydrodynamic force is converted to a torque, implemented by the electromagnetic torque, and the mass is converted to a rotary inertia to be represented by the rotor inertia of the machine adjusted with a flywheel if necessary.

Next to the buoy mass, a part of the hydrodynamic force can also be inserted physically in the setup. The linear added mass term of the radiation force, $F_{\text {rad, } 1}$ of Equation (8), is proportional with the buoy acceleration and is therefore referred to as the hydrodynamic inertia term. It can be seen as the inertia force due to a mass of water $m_{a, \infty}$ surrounding the buoy moving along with the buoy mass. When this force was to be calculated based on the derivative of a speed measurement in the setup, this would result in a noisy outcome. Therefore it is proposed to combine the added mass $m_{a, \infty}$ together with the buoy mass $m$ to be physically represented in the inertia of the wave emulator. Therefore the hydrodynamic force is split up in a part that is calculated, $F_{\text {hyd,calc }}$, and the linear added mass term of the radiation force $F_{\text {rad,1 }}$ which is physically represented. $F_{\text {hyd,calc }}$ is thus defined as

$$
F_{\text {hyd,calc }}=F_{\text {hyd }}-F_{\text {rad }, 1}=F_{\text {ex }}+F_{\text {res }}+F_{\text {rad }, 2} .
$$

Consequently the equation of motion can be written as

$$
\left(m+m_{a, \infty}\right) \frac{d^{2} z}{d t^{2}}=F_{\text {hyd,calc }}+F_{\text {PTO }}
$$

Fig. 5 schematically illustrates the representation of the floater and sea in the lab setup. In contrast with the cable that can only transfer forces in one direction, there is a fixed coupling between the PTO machine and the emulator machine. This is tackled by a torque transducer (HBM T22) in between the two shafts. The torque between the shafts is a measure for the cable force and is used to monitoring the cable force, and thus assess the ability of the PTO control to keep the cable under tension at all times. 


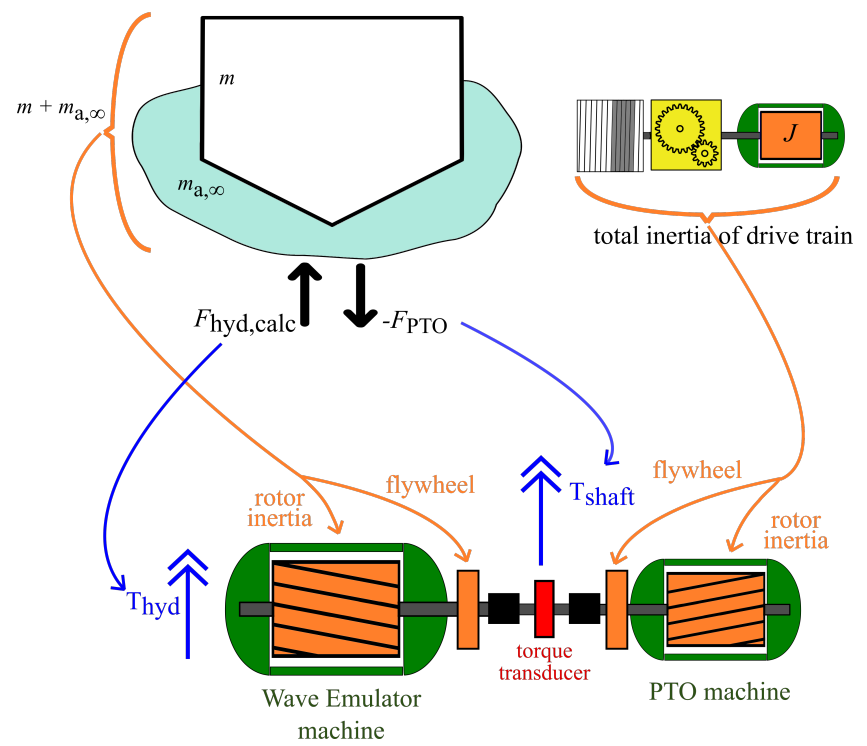

Figure 5: Schematic presentation of the correspondence between the floater at sea and PTO drive train with the setup

\subsubsection{Emulator torque and inertia}

The equivalent torque $T_{h y d}$ of the calculated hydrodynamic force is found using the same relationship between the PTO force and the shaft torque as in Equation (2):

$$
T_{\text {hyd }}=\frac{r}{R_{\mathrm{g}}} F_{\text {hyd,calc }}
$$

The equivalent rotary inertia for the buoy mass and added mass can be expressed as

$$
J_{\text {eq }}=\frac{\left(m_{\text {buoy }}+m_{\mathrm{a}, \infty}\right) r^{2}}{R_{\mathrm{g}}^{2}},
$$

considering that the masses can be seen as moving along the drum with radius $r$ and a gearbox with gear ratio $R_{\mathrm{g}}$ between the drum and machine shaft. Note that Equations (13) and 14 do not include any scaling, but only provide a rotary equivalent. 


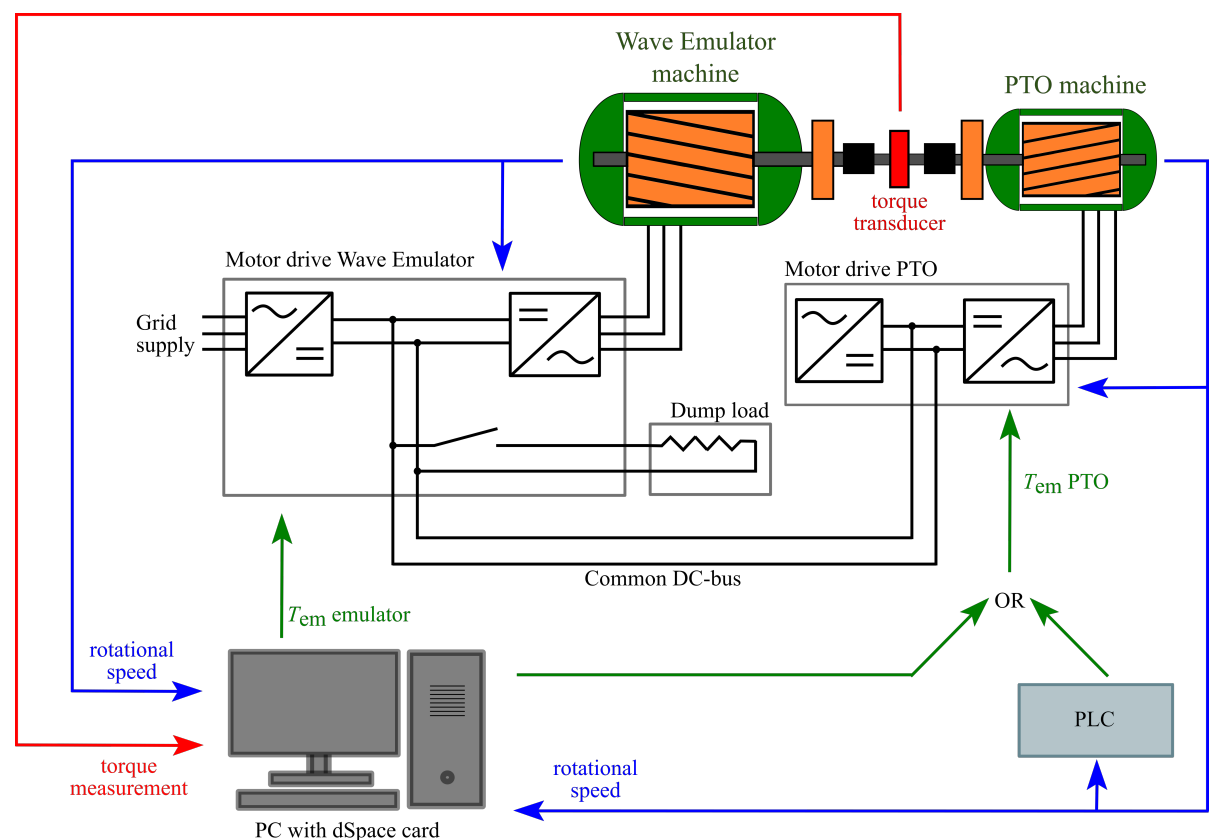

Figure 6: Schematic of the configuration of the setup

\subsection{Configuration}

The configuration of the setup is illustrated in Fig. 6. Each machine is connected by a frequency controlled motor drive and is provided with a forced cooling fan to assure ventilation at lower speeds. To optimise the resemblance with full scale, it is advised that the (scaled) PTO machine is of the same type as in the full-scale PTO. Next to the electrical machine, also the drive and other components need to be selected to have a close match. This has as additional advantage that drive settings can be tested and set in the lab environment and can afterwards easily be transferred to the real system.

\subsubsection{Control}

The calculation of Equation (11) is programmed in Matlab-Simulink ${ }^{\circledR}$, subsequently compiled to a dSPACE ${ }^{\circledR}$ rapid control prototyping card. The card has an embedded processor where the code is run to read inputs and control outputs. The program runs at $100 \mathrm{~Hz}$. As the model supposes that the floater 
moves in a pure heave motion and the cable is always kept taut, the drum speed and the length of the unwound cable is directly linked to the vertical speed and position of the floater. Thus the actual encoder position of the setup is read and used to calculate the buoy position and velocity. In this way, the measured signals are used to calculate the torque setpoint of the emulator in real-time which is sent to the drive by means of an analogue signal.

The implementation of the rapid control prototyping card provides great flexibility for extensive testing of the PTO and control algorithms. This type of card was chosen as it includes software enabling easy compilation of MatlabSimulink ${ }^{\circledR}$ code to the embedded processor of the card.

Also the PTO can be controlled by the $\mathrm{dSPACE}^{\circledR}$ environment, or as an alternative, by a programmable logic controller (PLC) identical as the one used in the WEC at sea, to test this part of the drive train.

\subsubsection{Grid connection of the setup}

As an energy saving measure, the two drives are connected on a common DC-bus, and only the wave emulator drive is supplied from the grid as shown in Fig. 6. At most of the instances in time when one of the machines acts as a motor, the other machine is be acting as a generator. Nevertheless, this behaviour is not always synchronous and depends on the applied PTO-control. Consequently, moments occur where one of the machines is generating at higher power than the other is consuming as motor. However, the rectifier of the drive is not regenerative, and a significant amount of energy is available in the rotary inertias, which the capacitors in the drives can not store. Therefore a dump load is necessary to dissipate excessive energy on the DC-bus when the actual generating power exceeds the actual consumed motor power.

The dimensioning of the dump load is WEC-device specific and can be calculated in simulation by summing the instantaneous powers of the PTO and emulator so that it can dump the peak generated resulting power.

By interconnecting the two drives on the DC-bus level, the consumed energy of the setup is reduced significantly: only the drive-train losses plus the dumped 
energy are taken from the grid.

\section{Scaling}

\subsection{Froude scaling}

To keep the installed power of the setup within the range of tens of kilowatts, a downscaling is necessary. The scaling proposed in this work consists of downscaling the complete wave energy converter and wave conditions, and subsequently represent this (virtual) scale model and scaled waves in the setup. Physical quantities of moving marine constructions can be scaled using Froude's Law [17, 18. This is a common method for physical scale model tests in wave flumes [19, 22, 23, and is also proposed for this dry lab setup because it enables easy transformation of data and parameters from the full scale design to the scale model, and backwards.

Next to the power, all hydrodynamic parameters to obtain the calculated hydrodynamic force need to be scaled as well. Including the timeseries of the exciting wave force, where not only the force is scaled, but also the time. Alternatively, timeseries of exciting wave force and hydrodynamic parameters can be generated based on the dimensions of the scaled floater. Both methods are equivalent.

The Froude scaling factor $\mu$ is the ratio between a length measurement of the full scale design $L_{F}$ and the model scale $L_{M}$.

$$
\mu=\frac{L_{F}}{L_{M}}
$$

The scale factors for other quantities according to Froude's Law can be found in Table 1. To dimension the setup, the relationships for torque and inertia are necessary:

$$
\begin{aligned}
& T_{\text {model }}=\frac{T_{\text {fullscale }}}{\mu^{4}} \\
& J_{\text {model }}=\frac{J_{\text {fullscale }}}{\mu^{5}}
\end{aligned}
$$




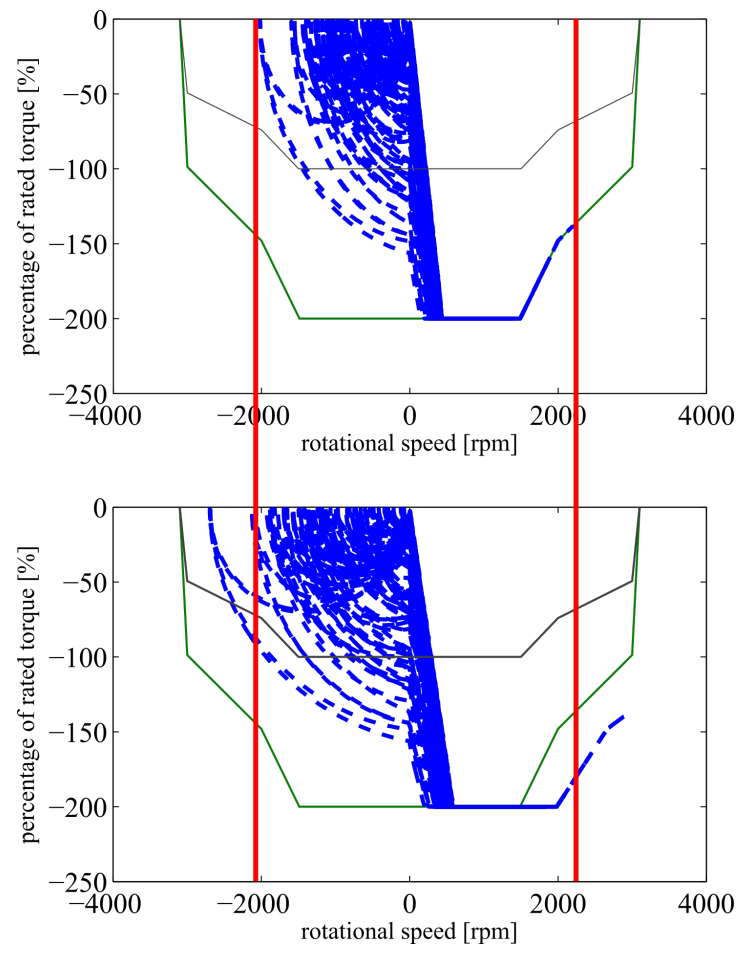

Figure 7: Applied torque-speed path (blue dashed) for the full scale (upper figure) and for the Froude scale model (lower figure) with the nominal (black) and $200 \%$ of nominal working range (green) 
Table 1: Froude Scaling Factors for Different Quantities

\begin{tabular}{lll}
\hline Quantity & Unit & Scale factor \\
\hline Length/distance & $\mathrm{m}$ & $\mu$ \\
Mass & $\mathrm{kg}$ & $\mu^{3}$ \\
Force & $\mathrm{N}$ & $\mu^{3}$ \\
Torque & $\mathrm{Nm}$ & $\mu^{4}$ \\
Time & $\mathrm{s}$ & $\mu^{0.5}$ \\
Velocity & $\mathrm{m} / \mathrm{s}$ & $\mu^{0.5}$ \\
Acceleration & $\mathrm{m} / \mathrm{s}$ & $\mu^{0}=1$ \\
Power & $\mathrm{W}$ & $\mu^{3.5}$ \\
Linear damping & $\mathrm{kg} / \mathrm{s}$ & $\mu^{2.5}$ \\
Rotational speed & $\mathrm{rpm}$ & $\mu^{-0.5}$ \\
Mass moment of inertia & $\mathrm{kg} . \mathrm{m}$ & $\mu^{5}$ \\
\hline
\end{tabular}

The consequence of Froude scaling is that the rotational speed of the machine in the scaled model is greater than in the full scale since

$$
n_{\text {model }}=n_{\text {fullscale }} \sqrt{\mu} \text {. }
$$

This is undesirable because the setup aimed at testing the PTO at equal normalized load conditions as at full scale. Fig. 7 displays that the load conditions change significantly due to the Froude scaling. Two load profiles - normalized torque vs. rotational speed - are plotted. The upper plot shows the load profile at full scale, the lower profile shows the load profile of the machine in a Froude scaled model where it is clear that the speed of all working points is increased significantly. To tackle this, a compensation method is proposed.

\subsection{Compensation by inertia to adapt speed after Froude scaling}

As a solution to the increase in rotational speed due to the Froude scaling, it is proposed to add inertia to the Froude scaled value of inertia of both the PTO 
side and the emulator side of the lab setup. By doing so, the speed decreases when applying an identical sequence of torque set-points to the setup. By using the right value of this additional inertia, the load curve corresponds to the full scale.

To appraise this value, $J_{\text {model }}$ and $\omega_{\text {model }}$ are defined as the Froude scaled inertia and angular velocity, and $J_{\text {comp }}$ and $\omega_{\text {comp }}$ as the speed-compensated inertia and angular velocity. The intention is to reduce the speed back to the values of the full scale, hence

$$
\omega_{\text {comp }}=\frac{\omega_{\text {model }}}{\sqrt{\mu}}
$$

As the torque sequence is kept equal in both situations, the following expression is valid:

$$
T=J_{\text {model }} \frac{d \omega_{\text {model }}}{d t}=J_{\text {comp }} \frac{d \omega_{\text {comp }}}{d t}
$$

and by inserting Equation (19) in Equation 20, the speed-compensated inertia is found:

$$
J_{\text {comp }}=J_{\text {model }} \sqrt{\mu}
$$

The compensated inertia should thus be $\sqrt{\mu}$ times larger than the Froude scaled value so that the normalized load curve of the setup corresponds with the normalized load curve of the full scale. This can be implemented by adding a flywheel to the inertia of the machine rotor to reach the compensated value, for both PTO machine and wave emulator machine.

\subsubsection{Consequences of the compensation towards the emulator control}

The calculation of the hydrodynamic torque for the emulator setpoint is based on the buoy position and speed. The computation of the buoy position and speed in the emulator control is based on the encoder data of the machine shaft and therefore needs to account for this compensation factor $\sqrt{\mu}$ too. The linear velocity of the Froude scale model for the case of the Wave Pioneer, 
consisting of a cable on a drum connected with a gearbox to the PTO machine, can then be expressed as:

$$
v_{\text {model }}=\frac{2 \pi r_{\text {mod }} n_{\text {comp }}}{60 R_{\mathrm{g}}} \sqrt{\mu}
$$

where $n_{\text {comp }}$ is the actual rotational speed in rpm of the compensated setup, $R_{\mathrm{g}}$ the gear ratio and $r_{\text {mod }}$ the radius of the drum in the scale model. Note that the gearbox ratio is not affected in the scaling.

\subsubsection{Consequences of the compensation towards interpreting the results}

Another implication is that the actual power $P_{\text {comp }}$ of the PTO machine no longer corresponds with the Froude scaled power $P_{\text {model }}$. If the power of the scale model is to be evaluated, it can be found as follows:

$$
P_{\text {model }}=P_{\text {comp }} \sqrt{\mu} \text {. }
$$

\section{Sizing the Setup}

\subsection{PTO torque}

The nominal power of the scaled PTO is chosen to be large enough to maintain close correspondence with the dynamic behaviour of the full scale PTO. A machine of $11 \mathrm{~kW}$ was chosen for this setup. The nominal torque of this machine is chosen as the starting point for the calculation of the Froude scale factor. This is justified by the fact that one of the main purposes of the setup is the assessment of the dynamic response of the PTO by keeping its relative load equal to the relative load of the full scale. However, other starting points for the scaling are possible. Thus, the scale factor is assigned as the ratio of the nominal torque of the full scale PTO $T_{\text {nomF }}$ and the nominal torque of the scaled model $T_{\text {nomm }}$ :

$$
\mu=\sqrt[4]{\frac{T_{\mathrm{nomF}}}{T_{\mathrm{nomM}}}}
$$


Based on this Froude scale factor, the dimensions of the virtual scaled buoy can be calculated using the factors in Table 1 . This scaled WEC is further referred to as 'scale model' or 'the model'. For the scale model the hydrodynamic parameters are calculated and time series for exciting wave forces are generated using WAMIT ${ }^{\circledR}$. These time series and scale model are the input for computer simulations for further dimensioning of the setup, and are used in the control of the setup.

\subsection{PTO inertia}

The complete mechanical drive train of a PTO in a real buoy contains more components (such as gearbox, drum) than the PTO side of the setup. Still, the inertia of all components need to be represented in the setup. Therefore the inertia of the full scale drive train is to be converted to an equivalent inertia at the machine shaft before a scaling can be performed. In Equation 25$] J_{\text {full }}$ is the equivalent inertia at the full scale machine shaft and $J_{\bmod }$ is the Froude scaled inertia for the model PTO:

$$
J_{\text {mod }}=\frac{J_{\text {full }}}{\mu^{5}} .
$$

To find the required value for a setup running at equal speed as the full scale, the compensation of Equation (21) needs to be added:

$$
J_{\text {compPTO }}=\frac{J_{\text {Fpto }}}{\mu^{4.5}}
$$

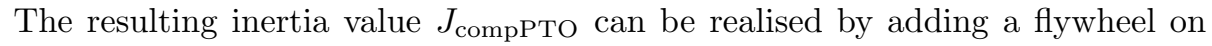
the shaft of the PTO machine so that the sum of the rotor inertia and flywheel

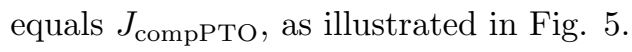

\subsection{Wave Emulator torque}

In most of the WEC designs the hydrodynamic forces exceed the maximum available PTO force at many instances, consequently the installed power of the emulator machine needs to be higher than the PTO machine. 
The hydrodynamic force is reproduced by the electromechanical torque of the emulator machine. To appoint the required emulator machine, computer simulations [19] of the hydrodynamic movement of the virtual WEC are performed. The total hydrodynamic force $F_{\text {hyd,calc }}$ the scale model would encounter is calculated in time domain simulations for the six sea states. The force is thereafter translated to the torque of the emulator machine using Equation 13 which accounts for all PTO drive train transmission ratios until the shaft of the PTO machine, such as drum and gearbox. Note that the scaled drum radius must be used. Next, the torque (T) is plotted versus the rotational speed (n) at each moment to define the needed working region for the Wave Emulator electrical machine.

These torque-speed $(T-n)$ plots permit a graphical determination of the required power for the emulator machine. The choice of the appropriate machine can then be done by fitting the working region across the most demanding load curve. As new control strategies might be more demanding and arouse higher hydrodynamic forces or higher speeds, it is advised to take sufficient margin during the sizing.

As the movement in our case is intermittent, good engineering practice allows to use a machine up to the maximum allowed torque of the machine specifications, as long as the RMS torque remains below the rated torque. This practice reduces the necessary rated power by $50 \%$ when the maximum allowed torque is $200 \%$ of the nominal. Moreover, as the plots in Fig. 8 do not show high torque needs at higher speeds, usage of super-rated speed region seems appropriate to realize a second reduction of installed power. Thus a machine with a rated speed of $1500 \mathrm{rpm}$ is chosen and used up to $3000 \mathrm{rpm}$. The machine is used up to $200 \%$ of its rated torque. The same measures have earlier been adopted in the dimensioning of the (full scale) Wave Pioneer's PTO [24].

In Fig. 8 the selected working region of $200 \%$ of rated torque and speed for three different machine sizes $(18.5 \mathrm{~kW}, 22 \mathrm{~kW}$ and $30 \mathrm{~kW})$ have been fitted above the load plots of the three most demanding sea states.

To provide ample margin in higher loads and increase experimental potential 

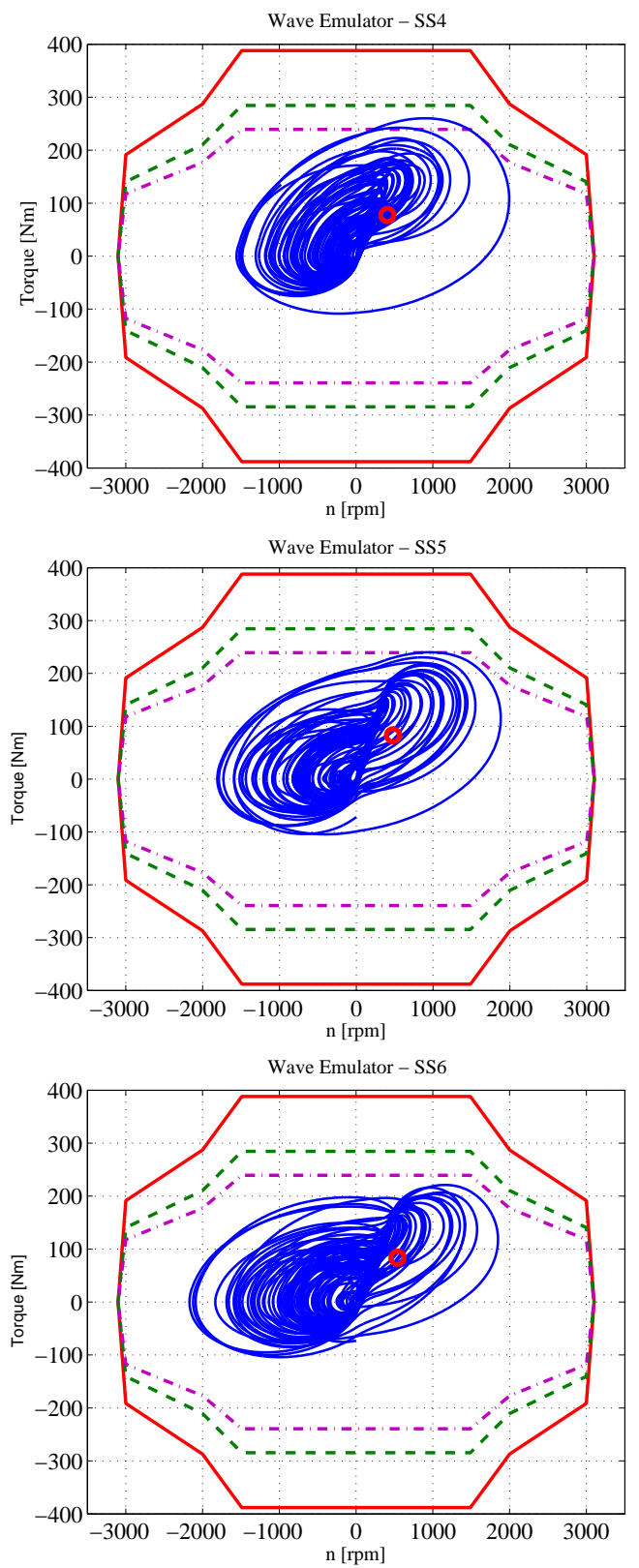

Figure 8: Torque-speed $200 \%$ working regions for three different machine sizes fitted above the most demanding load plots. Machine sizes of $18.5 \mathrm{~kW}$ (purple -.), $22 \mathrm{~kW}$ (green -) and $30 \mathrm{~kW}$ (red solid line). Load curves in blue solid line. 
towards the future, for our setup a $30 \mathrm{~kW} 1500 \mathrm{rpm}$ rated induction machine was chosen. The red circle in Fig. 8 points at the RMS torque, which is in all cases lower than the nominal torque.

The inertia of the rotor can be decisive in the machine choice, as it should not exceed the value of the compensated reproduced inertia corresponding with the buoy mass and added mass. The calculation of this scaling is found in the next paragraph.

\subsection{Wave Emulator inertia}

As introduced earlier, a scaling similarity is achieved by including the buoy's mass $m_{\text {buoy }}$ and added mass $m_{a, \infty}$ in the rotational inertia of the emulator machine. This avoids inertia compensation calculations with noisy acceleration signals.

In order to do so, the sum of the floater's mass and added mass must be converted to an equivalent inertia $J_{\text {eq }}$ at the shaft of the PTO. In case of the Wave Pioneer, consisting of a cable on a drum connected with a gearbox to the PTO machine, this is done using the expression of Equation (14). Assuming this is done for the full scale floater, the inertia for the emulator side of the setup is scaled analogous to the calculation of the PTO inertia. Hence, the emulator's rotary inertia for a setup running at equal speed as the full scale is:

$$
J_{\text {comp emu }}=\frac{J_{\text {eq }}}{\mu^{4.5}}
$$

As for the PTO part, this equivalent inertia $J_{\text {comp emu }}$ can be established with the total rotational inertia on the emulator side of the setup, i.e. machine rotor, coupling and half of the torque transducer's inertia, expanded with a flywheel to match the calculated value.

\subsection{Overview of lab setup electrical machines and drives}

Table 2 collects the specifications of the main components of the setup following the design concept described in this paper. The electrical machines are 
intermittently used up to $200 \%$ of their nominal power. To ensure this overrated operation, the drives are dimensioned to a rated power of at least $200 \%$ of the rated machine power since the overloading ability of the drives is much smaller than machines due to the limited thermal inertia of the power electronics. The final attained power for the drives was the first available power rating matching or exceeding the maximum used motor power.

Table 2: Overview of the setup's main specifications

\begin{tabular}{lcc}
\hline & PTO & Wave Emulator \\
\hline Electrical machine & & \\
Rated power & $11 \mathrm{~kW}$ & $30 \mathrm{~kW}$ \\
Maximum power & $22 \mathrm{~kW}$ & $60 \mathrm{~kW}$ \\
Rated torque & $71.5 \mathrm{Nm}$ & $194 \mathrm{Nm}$ \\
Maximum torque & $143 \mathrm{Nm}$ & $388 \mathrm{Nm}$ \\
Rated speed & $1500 \mathrm{rpm}$ & $1500 \mathrm{rpm}$ \\
Maximum speed & $3000 \mathrm{rpm}$ & $3000 \mathrm{rpm}$ \\
Variable frequency drive & & \\
Rated power & $22 \mathrm{~kW}$ & $75 \mathrm{~kW}$ \\
\hline
\end{tabular}

\section{Validation of the Emulator}

To verify the accuracy of the wave emulator control, experimental results of the lab setup have been compared with simulation [19] results. It is considered that the emulation of the WEC motion can be approved if the resulting speed curves of the setup and simulation correlate well when run with the same wave excitation force time series and same PTO force.

Normally, the PTO force in the simulation is calculated following the control method of [3] and is thus proportional to the actual speed and acceleration. To focus on the accuracy of the emulator, for this validation the calculation of the PTO force has been omitted in an adapted simulation model. Instead, the 
measured cable force of the setup (represented by the torque measurement) is used as input for the calculation of the equation of movement of the WEC. The time series of the wave exciting force for the simulation is equal as in the setup to use as a simulation input. Using this method, the virtual WEC in the simulation experiences the same forces as in the setup.

The analysis is done with the actual rotational speed of the setup, meaning that the speed of the simulation is recalculated to the corresponding compensated speed as defined in Equation 19.

The comparison has been carried out for all six sea states for a time window of approximately 8 minutes. For each sea state the WEC speed has been processed and examined by means of the PTO rpm. The rotational speeds of setup and simulation together with the rpm error from Equation 28 have been plotted in Fig. 9 .

$$
\text { error }_{\mathrm{rpm}}=\text { rpm }_{\mathrm{setup}}-\text { rpm }_{\mathrm{sim} \_c o m p}
$$

Subsequently histograms of the relative error show the distribution of the relative error magnitudes in Fig. 10 .

$$
\text { Rel_error }_{\mathrm{rpm}}=\frac{r p m_{\mathrm{setup}}-r p m_{\mathrm{sim} \_c o m p}}{r p m_{\mathrm{sim} \_c o m p}}
$$

Fig. 9 zooms in on 100 s of the results. It indicates that the rpm error is relatively high in the lowest sea state, SS1. Once the load increases at higher sea states, the rpm error decreases resulting in a setup speed curve that approximates the simulated one. The absorbed power in SS1 is too low to have a net electrical energy production, consequently the difference in speed curve for this sea state can be ignored, because the sea state is irrelevant to draw any conclusions about the PTO. From SS2 on, the rpm error reduces significantly and the speed curves show a satisfying match.

The accuracy of the emulator depends on a number of factors. Firstly, the control is an open loop torque control and any deviation influences the setup's 


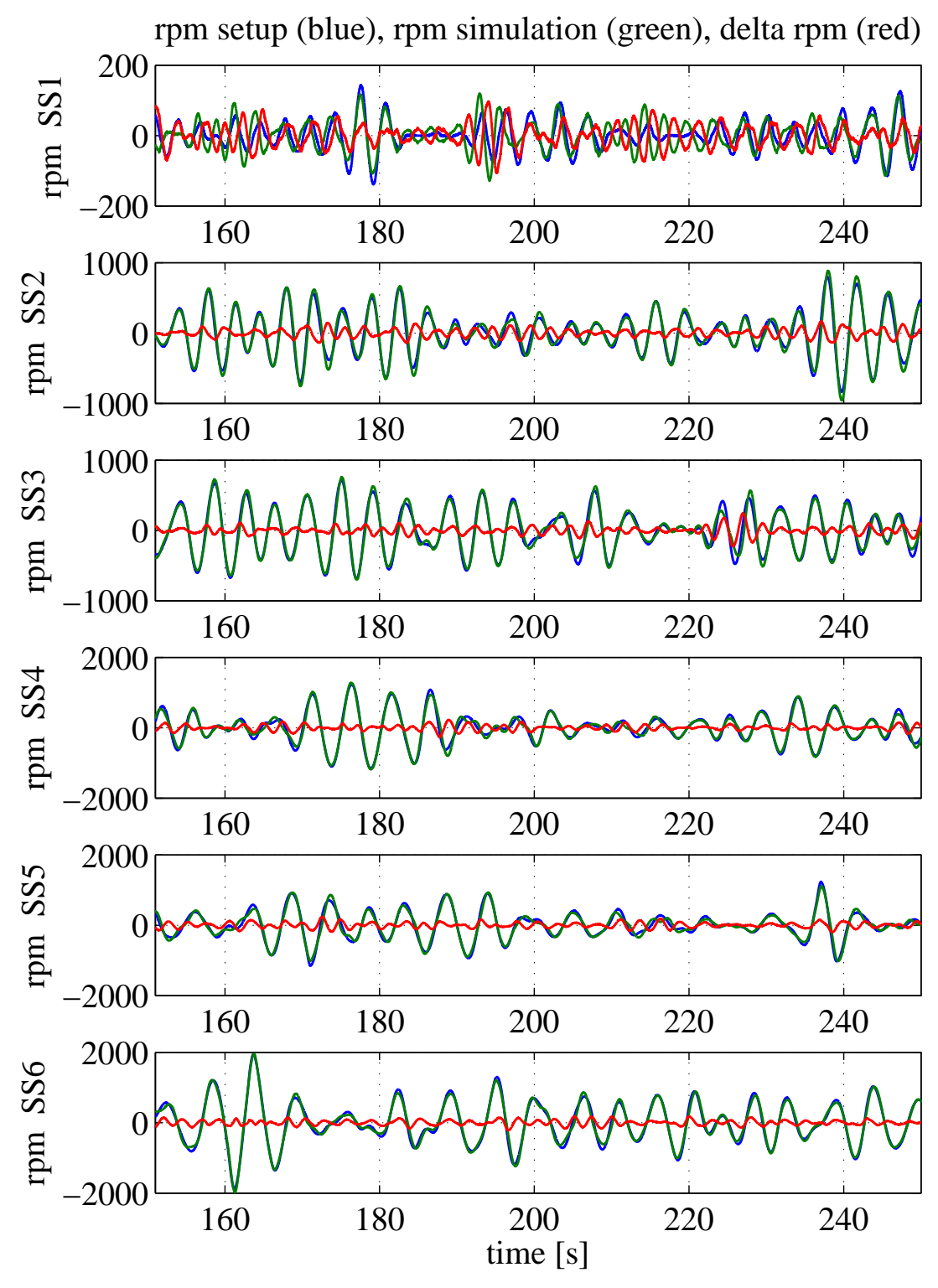

Figure 9: Plots of 100s of the rotational speed of setup (blue), simulation (green) and the speed error (red) for sea state 1 up to 6 

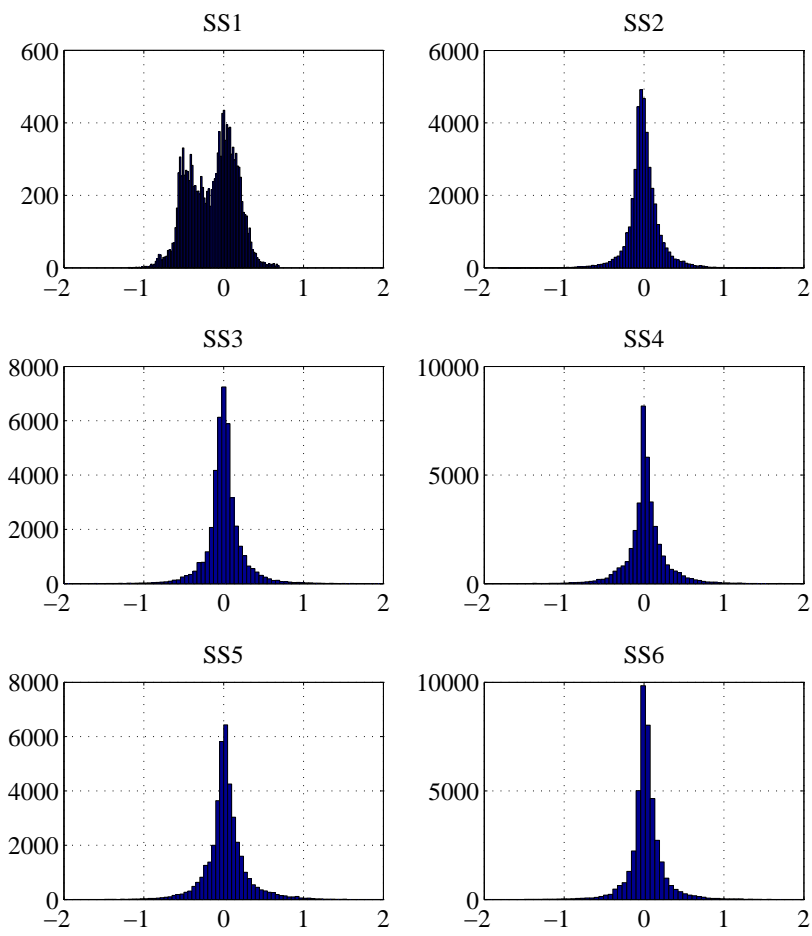

Figure 10: Histograms number of occurrence vs. relative rpm error per sea state

speed. When comparing against the simulations, it should be kept in mind that the friction of the bearings is not taken into account because measurements of the friction with peaks of $2 \mathrm{Nm}$ were categorised as negligible compared to peak set-point torques of up to $300 \mathrm{Nm}$. Another potential cause of deviation is the possible difference between the final rotational inertia in the setup and the value from the datasheets due to minor modifications to the motor shaft (removal of fans and adding encoders) and couplings. This discrepancy in inertia is rather small and a more precise value could not be determined experimentally.

The convergence in the correlation can be seen in the histograms of the relative error from Equation (29) in Fig. 10. 


\section{Conclusions}

In this paper, a wave emulator PTO test setup is presented. A lab setup should be a tool for performing dynamic response tests and long duration tests for a power take-off system as if it was in a wave energy converter at sea.

The presented setup succeeds in providing an environment with equal normalized load conditions using a Froude scale model. This has been realized by implementing the hydrodynamic model of the floater in the setup. To avoid calculations with noisy acceleration signals, all mass terms of the hydrodynamic model have been represented physically as rotary inertias in the wave emulator. The undesired consequence of the increased rotational speed after Froude scaling has been dealt with by adding extra inertia to the setup.

The experimental results of the wave emulator setup have been compared to simulation using equal power take-off forces on the scaled model. The comparison showed good correlation for the motion of the floater for the significant sea states. This makes the proposed Wave Emulator a valuable tool in the development of power take-off systems for wave energy converters.

\section{Acknowledgement}

A part of the work presented in this paper was carried out during the FlanSea project, financed by the Agency for Innovation by Science and Technology in Flanders (IWT) and the industrial partners DEME Blue Energy, Cloostermans, Spiromatic, Port of Oostende, Electrawinds and Contec.

\section{References}

[1] The flansea website (flanders electricity from the sea), URL: http://www. flansea.eu/, accessed date: December 2015.

[2] A. F. de O. Falcão, Wave energy utilization: A review of the technologies, Renewable and Sustainable Energy Reviews 14 (3) (2010) 899 - 918. doi: 10.1016/j.rser.2009.11.003. 
[3] M. Vantorre, R. Banasiak, R. Verhoeven, Modelling of hydraulic performance and wave energy extraction by a point absorber in heave, Applied Ocean Research 26 (1-2) (2004) 61-72. doi:10.1016/j.apor.2004.08. 002 .

[4] V. Stratigaki, P. Troch, T. Stallard, D. Forehand, J. Kofoed, M. Folley, M. Benoit, A. Babarit, J. Kirkegaard, Wave basin experiments with large wave energy converter arrays to study interactions between the converters and effects on other users in the sea and the coastal area, Energies 7 (2) (2014) 701-734. doi:10.3390/en7020701.

[5] V. Stratigaki, P. Troch, T. Stallard, D. Forehand, M. Folley, J. P. Kofoed, M. Benoit, A. Babarit, M. Vantorre, J. Kirkegaard, Sea-state modification and heaving float interaction factors from physical modelling of arrays of wave energy converters, Journal of Renewable and Sustainable Energy 7 (6) (2015) 061705. doi:10.1063/1.4938030.

[6] V. Stratigaki, Experimental study and numerical modelling of intra-array interactions and extra-array effects of wave energy converter arrays., Ph.D. thesis, Ghent University, Faculty of Engineering and Architecture, Ghent, Belgium (2014).

[7] A. Van de Sijpe, Development of a point absorber wave energy converter: realisation of power take-off, optimisation of geometry and installation techniques, Master's thesis, Ghent University (2012).

[8] Y. Hong, R. Waters, C. Bostrom, M. Eriksson, J. Engstrom, M. Leijon, Review on electrical control strategies for wave energy converting systems, Renewable \& Sustainable Energy Reviews 31 (2014) 329-342. doi:10. 1016/j.rser.2013.11.053.

[9] A. F. d. O. Falcão, P. E. R. Pereira, J. C. C. Henriques, L. M. C. Gato, Hydrodynamic simulation of a floating wave energy converter by a u-tube rig for power take-off testing, Ocean Engineering 37 (14-15) (2010) 12531260. doi:10.1016/j.oceaneng.2010.05.007. 
[10] N. J. Baker, M. A. Mueller, L. Ran, P. J. Tavner, S. McDonald, Development of a linear test rig for electrical power take off from waves, Journal of Marine Engineering and Technology 6 (2) (2007) 3-15. doi: $10.1080 / 20464177.2007 .11020201$

[11] M. Blanco, M. Lafoz, L. Garcia Tabares, Laboratory tests of linear electric machines for wave energy applications with emulation of wave energy converters and sea waves, in: 14th European Conf. Power Electronics and Applications, 2011.

[12] R. Henderson, Design, simulation, and testing of a novel hydraulic power take-off system for the pelamis wave energy converter, Renewable Energy 31 (2) (2006) 271-283. doi:10.1016/j.renene.2005.08.021.

[13] J. Rea, J. Kelly, R. Alcorn, D. OSullivan, Development and operation of a power take off rig for ocean energy research and testing, in: Proc. 2011 European Wave and Tidal Energy Conf., 2011.

[14] J. Henriques, R. Gomes, L. Gato, A. Falco, E. Robles, S. Ceballos, Testing and control of a power take-off system for an oscillating-water-column wave energy converter, Renewable Energy 85 (2016) 714 - 724. doi:10.1016/ j.renene.2015.07.015.

[15] J. Henriques, L. Gato, A. Falco, E. Robles, F.-X. Fa, Latching control of a floating oscillating-water-column wave energy converter, Renewable Energy 90 (2016) 229 - 241. doi:10.1016/j.renene.2015.12.065.

[16] J. F. Gaspar, M. Kamarlouei, A. Sinha, H. Xu, M. Calvrio, F.-X. Fa, E. Robles, C. G. Soares, Speed control of oil-hydraulic power take-off system for oscillating body type wave energy converters, Renewable Energy 97 (2016) 769 - 783. doi:10.1016/j.renene.2016.06.015.

[17] W. Froude, Observations and Suggestions on the Subject of Determining by Experiment the Resistance of Ships, The Papers of William Froude, 1810-1879, Transactions INA, 1955. 
[18] D. Vassalos, Physical modelling and similitude of marine structures, Ocean

n Engineering 26 (2) (1998) 111 - 123. doi:10.1016/S0029-8018(97) $10004-\mathrm{X}$

[19] G. De Backer, M. Vantorre, K. De Beule, C. Beels, J. De Rouck, Experimental investigation of the validity of linear theory to assess the behaviour of a heaving point absorber at the belgian continental shelf, ASME 28th International Conference on Ocean, Offshore and Arctic Engineering (OMAE 2009), Vol. 4 (2009) 1013-1020.

[20] W. Cummins, The impulse response function and ship motions, Schiffstechnik 9 (1962) 101-109.

[21] G. Duclos, A. H. Clement, G. Chatry, Absorption of outgoing waves in a numerical wave tank using a self-adaptive boundary condition, International Journal of Offshore and Polar Engineering 11 (3) (2001) 168-175.

[22] M. Durand, A. Babarit, B. Pettinotti, O. Quillard, J. Toularastel, A. H. Clment, Experimental validation of the performances of the searev wave energy converter with real time latching control, in: 7th European Wave and Tidal Energy conference, 2007.

[23] R. Gomes, J. Henriques, L. Gato, A. F. ao, Wave power extraction of a heaving floating oscillating water column in a wave channel, Renewable Energy 99 (2016) 1262 - 1275. doi:10.1016/j.renene.2016.08.012.

[24] K. De Koker, G. Crevecoeur, B. Meersman, M. Vantorre, L. Vandevelde, A power take-off and control strategy in a test wave energy converter for a moderate wave climate, Renewable Energy and Power Quality Journal 14 (May 2016). 